\title{
Macular neovascularization in AMD, CSC and best vitelliform macular dystrophy: quantitative OCTA detects distinct clinical entities
}

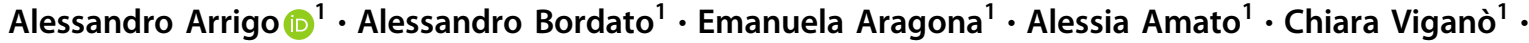 \\ Francesco Bandello ${ }^{1}$ Maurizio Battaglia Parodi ${ }^{1}$
}

Received: 15 June 2020 / Revised: 16 December 2020 / Accepted: 7 January 2021 / Published online: 25 January 2021

(c) The Author(s), under exclusive licence to The Royal College of Ophthalmologists 2021

\begin{abstract}
Background To perform a quantitative optical coherence tomography (OCT) angiography (OCTA) analysis of macular neovascularization (MNV) secondary to age-related macular degeneration (AMD), central serous chorioretinopathy (CSC) and best vitelliform macular dystrophy (BVMD), with the aim of highlighting quantitative features indicating different clinical entities.

Methods Study design: prospective, interventional. We recruited patients affected by AMD, CSC or BVMD, complicated by naive MNV. All patients underwent complete ophthalmologic examination and multimodal imaging. They were treated with anti-VEGF injections, following a pro-re-nata regimen. The ensuing follow-up lasted 1 year. Quantitative dye-based angiography, OCT, and OCTA parameters were analysed to obtain cutoff values able to distinguish two clinically different patient subgroups for each retinal disease. The main outcome measures were best-corrected visual acuity (BCVA), central macular thickness, vessel density of superficial, deep and choriocapillaris plexa, vessel tortuosity (VT) of MNV, vessel dispersion of MNV, number of injections, MNV/leakage ratio, MNV size, speckled fluorescence, and outer retinal atrophy. Results Ninety-eight eyes affected by MNV (98 patients) were analysed. These included 66 eyes affected by AMD, 18 displaying CSC, and 14 eyes with BVMD. BCVA was alike in the three groups, both at baseline and after 1 year $(p>0.05)$. An MNV VT cutoff of 8.40 at baseline detected two patient subgroups differing significantly in terms of morpho-functional features, found both at baseline and at the end of the follow-up.

Conclusions Quantitative OCTA suggested that the MNV's VT might be able to provide a better characterization of two different morpho-functional manifestations in AMD, CSC and BVMD.
\end{abstract}

\section{Introduction}

Choroidal neovascularization is a common complication of several retinal diseases, occurring as the consequence of abnormal release of vascular endothelial growth factor (VEGF) from the retinal pigment epithelium (RPE) and Muller cells [1]. A panel of experts recently

Supplementary information The online version contains supplementary material available at https://doi.org/10.1038/s41433021-01396-2.

Alessandro Arrigo

alessandro.arrigo@hotmail.com

1 Department of Ophthalmology, IRCCS San Raffaele Hospital, Vita-Salute University, Milan, Italy suggested updating the nomenclature with regard to agerelated macular degeneration (AMD), including a fresh definition of macular neovascularization (MNV) [2]. MNV can be studied by means of conventional dye-based angiography as well as structural optical coherence tomography (OCT), both of which provide useful information regarding MNV localization and the amount of exudation. More recently, OCT angiography (OCTA) has considerably increased our knowledge of the morphological features of MNV [3-6]. The adoption of quantitative OCTA-based metrics has further improved our understanding of the anatomical and clinical outcomes of eyes affected by MNV, providing clinically useful quantitative cutoffs to predict the final outcome [5, 6]. However, these types of approach have only been applied in the context of patients suffering from age-related macular degeneration (AMD). 
It was our aim in the present study to investigate the application of a OCTA quantitative metric related to the vessel tortuosity of the MNV in macular diseases other than AMD. In particular, we analysed MNV secondary to central serous chorioretinopathy (CSC) and best vitelliform macular dystrophy (BVMD), comparing the two forms with MNV related to AMD to determine whether each MNV has distinctive quantitative OCTA characteristics.

\section{Methods}

The study was designed as a prospective, interventional case series. Consecutive patients affected by AMD, CSC or BVMD, complicated by naive MNV, were recruited at the Ophthalmology Unit of San Raffaele Hospital, Milan, Italy. The study was approved by the Ethical Committee of the Vita-Salute San Raffaele University in Milan and conducted in accordance with the Declaration of Helsinki. All patients gave signed, informed consent before the examinations. All MNVs were treated with Ranibizumab $0.5 \mathrm{mg}$ intravitreal injections, starting with a loading dose of three-monthly injections, followed by further treatments in accordance with the pro-re-nata (PRN) regimen over a planned 1-year follow-up. MNVs secondary to CSC underwent combined treatment consisting of half-dose photodynamic therapy (PDT) and anti-VEGF injections.

The inclusion criteria were naive AMD-, CSC- or BVMDrelated $\mathrm{MNV}$, classified as type 1, type 2 and mixed type 1 and 2, on the basis of fluorescein angiography (FA) and indocyanine green angiography (ICGA) (Spectralis HRA + OCT; Heidelberg Engineering, Heidelberg, Germany).

We excluded all the other types of neovascular lesions, such as polypoidal choroidal vasculopathy and retinal angiomatous proliferation, owing to the poor OCTA image quality of these lesions. Moreover, we also excluded eyes displaying pigment epithelium detachment $>200 \mu \mathrm{m}$ for the same reason. Further exclusion criteria were high media opacities, any other ophthalmological disorder, ophthalmological surgery within the last 6 months, or any systemic condition potentially affecting the analyses. The refractive error range was between $-3 \mathrm{D}$ and $+1.5 \mathrm{D}$.

All patients underwent complete ophthalmological examination, including BCVA measurement using standard ETDRS charts, slit-lamp biomicroscopic examination of anterior and posterior segments, and Goldmann applanation tonometry.

Structural OCT acquisitions included raster, radial, and dense scans, with a high number of frames (ART $>25$ ) and enhanced depth imaging (EDI). We measured central macular thickness (CMT), subfoveal choroidal thickness (CT), Haller layer thickness (HLT) and Sattler layer thickness (SLT), at baseline and at the 1-year follow-up.
OCTA images were obtained using a swept source OCT DRI Topcon Triton (Topcon Corporation, Tokyo, Japan). OCTA scans included high-resolution $3 \times 3 \mathrm{~mm}$ and $4.5 \times 4.5$ $\mathrm{mm}$ acquisitions. Only high-quality images, evaluated by Topcon Imaging Quality factor $>80$, were considered. We isolated superficial (SCP), deep (DCP) and choriocapillaris (CC) plexa from the $4.5 \times 4.5 \mathrm{~mm}$ acquisitions, as well as the MNV network from $3 \times 3 \mathrm{~mm}$ OCTA reconstructions.

We obtained vascular plexa vessel density (VD), MNV network VT and vessel dispersion (Vdisp) values by performing the same post-processing steps already described in our previous paper [5].

As a preliminary step, all the quantitative metrics needed to be binarized through a "mean" automatic threshold. VD was calculated as the ratio of white to black pixels; the foveal avascular zone was manually segmented and excluded from the calculation. VT and Vdisp of the MNV required image skeletonization through a Fiji software (https://imagej.net/Fiji/Downloads) pipeline named "Skeletonize". The "Analyze skeleton" tool was then used to extract VT, while the "Directionality" tool was adopted to extract Vdisp for each MNV.

VT can be defined as the ratio of the shortest pathway to the straight-line length, and is intended as a measure of the perfusion ratio of a given vascular network. VT was extracted by the "Analyze skeleton" tool by measuring the Euclidean distance along each skeletonized line, and defined as the ratio between each line's length and the shortest linear distance between the initial and the final point $[5,6]$.

Vdisp can be defined as the measure of the space-filling capacity of a given vascular network and of its degree of disorganization. In particular, Vdisp pipeline works assuming that each vessel's direction can be studied through a Gaussian function. The measure of data dispersion, defined as the standard deviation from the Gaussian distribution, may express the degree of disorganization in the same network, in our case MNV [5, 6].

OCTA quantitative analyses were performed considering only baseline reconstructions. On the basis of previous experience of MNV OCTA assessment [5, 6], we applied an MNV VT cutoff of 8.40 to differentiate two clinically significant MNV groups of patients: Group $1(\mathrm{VT}<8.40)$ and Group 2 (VT > 8.40).

Other parameters analysed were the leakage area (defined as the extension of the region affected by leakage $5 \mathrm{~min}$ after the fluorescein dye injection), the MNV area (measured on ICGA), and the retinal quadrants of speckled fluorescence (SF) (defined as multiple punctate spots of hyperfluorescence observed $5 \mathrm{~min}$ after the fluorescein dye injection). SF was measured by dividing the posterior pole into four quadrants (two temporal and two nasal) and by considering only the quadrants involved, using the center of 
Table 1 Clinical and imaging data in MNV secondary to AMD, CSC and BVMD.

\begin{tabular}{|c|c|c|c|c|c|c|}
\hline \multicolumn{7}{|l|}{ Clinical and imaging data in MNV } \\
\hline \multirow[t]{2}{*}{ Parameter } & \multirow[t]{2}{*}{ MNV type } & \multirow[t]{2}{*}{ Number } & \multirow[t]{2}{*}{ Mean \pm STD } & \multicolumn{3}{|c|}{$p$ value } \\
\hline & & & & 1 vs 2 & 1 vs 3 & 2 vs 3 \\
\hline \multirow[t]{3}{*}{ Age } & AMD & 1 & $77 \pm 7$ & $p<0.001 *$ & $p<0.001 *$ & $p<0.001 *$ \\
\hline & $\mathrm{CSC}$ & 2 & $61 \pm 11$ & & & \\
\hline & BVMD & 3 & $21 \pm 12$ & & & \\
\hline \multirow[t]{3}{*}{ Speckled fluorescence (quadrants) } & AMD & 1 & $1.3 \pm 1$ & $p<0.001^{*}$ & $p<0.001^{*}$ & $p<0.001^{*}$ \\
\hline & $\mathrm{CSC}$ & 2 & $1.8 \pm 1$ & & & \\
\hline & BVMD & 3 & $0 \pm 0$ & & & \\
\hline \multirow[t]{3}{*}{ Leakage area } & AMD & 1 & $4.2 \pm 4.1$ & $p>0.05$ & $p<0.001^{*}$ & $p<0.001^{*}$ \\
\hline & $\mathrm{CSC}$ & 2 & $3.8 \pm 1.9$ & & & \\
\hline & BVMD & 3 & $2.6 \pm 1.3$ & & & \\
\hline \multirow[t]{3}{*}{ MNV area } & AMD & 1 & $2.9 \pm 3.4$ & $p>0.05$ & $p<0.001^{*}$ & $p>0.05$ \\
\hline & $\mathrm{CSC}$ & 2 & $1.5 \pm 1.4$ & & & \\
\hline & BVMD & 3 & $0.9 \pm 0.5$ & & & \\
\hline \multirow[t]{3}{*}{ MNV/leakage ratio } & AMD & 1 & $0.7 \pm 0.3$ & $p<0.001 *$ & $p<0.001^{*}$ & $p>0.05$ \\
\hline & $\mathrm{CSC}$ & 2 & $0.4 \pm 0.2$ & & & \\
\hline & BVMD & 3 & $0.4 \pm 0.2$ & & & \\
\hline \multirow[t]{3}{*}{ LogMAR BCVA baseline } & AMD & 1 & $0.50 \pm 0.61$ & $p>0.05$ & $p>0.05$ & $p>0.05$ \\
\hline & $\mathrm{CSC}$ & 2 & $0.33 \pm 0.18$ & & & \\
\hline & BVMD & 3 & $0.24 \pm 0.18$ & & & \\
\hline \multirow[t]{3}{*}{ LogMAR BCVA 1-year } & AMD & 1 & $0.31 \pm 0.29$ & $p>0.05$ & $p>0.05$ & $p>0.05$ \\
\hline & $\mathrm{CSC}$ & 2 & $0.25 \pm 0.19$ & & & \\
\hline & BVMD & 3 & $0.30 \pm 0.28$ & & & \\
\hline \multirow[t]{3}{*}{ CMT Baseline } & AMD & 1 & $408 \pm 105$ & $p>0.05$ & $p>0.05$ & $p>0.05$ \\
\hline & $\mathrm{CSC}$ & 2 & $366 \pm 107$ & & & \\
\hline & BVMD & 3 & $407 \pm 87$ & & & \\
\hline \multirow[t]{3}{*}{ CMT 1-year } & AMD & 1 & $348 \pm 76$ & $p<0.001 *$ & $p<0.001 *$ & $p<0.001 *$ \\
\hline & $\mathrm{CSC}$ & 2 & $228 \pm 49$ & & & \\
\hline & BVMD & 3 & $310 \pm 59$ & & & \\
\hline \multirow[t]{3}{*}{ N. intravitreal injections } & AMD & 1 & $7 \pm 2$ & $p>0.05$ & $p<0.001^{*}$ & $p<0.001^{*}$ \\
\hline & $\mathrm{CSC}$ & 2 & $6 \pm 3$ & & & \\
\hline & BVMD & 3 & $1 \pm 2$ & & & \\
\hline \multirow[t]{3}{*}{ VT MNV baseline } & AMD & 1 & $8.4 \pm 1.3$ & $p>0.05$ & $p<0.001^{*}$ & $p<0.001^{*}$ \\
\hline & $\mathrm{CSC}$ & 2 & $8.0 \pm 1.2$ & & & \\
\hline & BVMD & 3 & $7.5 \pm 1.2$ & & & \\
\hline \multirow[t]{3}{*}{ Vdisp MNV baseline } & AMD & 1 & $29.86 \pm 13.29$ & $p<0.001^{*}$ & $p>0.05$ & $p<0.001^{*}$ \\
\hline & $\mathrm{CSC}$ & 2 & $20.26 \pm 3.88$ & & & \\
\hline & BVMD & 3 & $31.71 \pm 13.90$ & & & \\
\hline \multirow[t]{3}{*}{ VD SCP baseline } & AMD & 1 & $0.37 \pm 0.02$ & $p>0.05$ & $p>0.05$ & $p>0.05$ \\
\hline & $\mathrm{CSC}$ & 2 & $0.38 \pm 0.03$ & & & \\
\hline & BVMD & 3 & $0.38 \pm 0.03$ & & & \\
\hline \multirow[t]{3}{*}{ VD DCP Baseline } & AMD & 1 & $0.36 \pm 0.02$ & $p>0.05$ & $p<0.001^{*}$ & $p<0.001 *$ \\
\hline & CSC & 2 & $0.35 \pm 0.02$ & & & \\
\hline & BVMD & 3 & $0.38 \pm 0.02$ & & & \\
\hline \multirow[t]{3}{*}{ VD CC Baseline } & AMD & 1 & $0.45 \pm 0.03$ & $p>0.05$ & $p>0.05$ & $p>0.05$ \\
\hline & $\mathrm{CSC}$ & 2 & $0.46 \pm 0.04$ & & & \\
\hline & BVMD & 3 & $0.47 \pm 0.03$ & & & \\
\hline
\end{tabular}

the $\mathrm{MNV}$ as a reference point. We calculated the MNV/ leakage ratio to quantify the amount of leakage in relation to the size of the MNV and to have a parameter associated with the activity of the MNV.

Two independent graders performed all the measurements $(\mathrm{AA}, \mathrm{AB})$, by calculating each of these parameters at least twice in order to test reproducibility and repeatability. The interclass correlation coefficient (ICC) was measured to assess the extent of agreement between the two graders.

The statistical analyses were performed using the SPSS software package (SPSS, Chicago, IL, USA). Age, gender, and baseline features were considered as fixed factors. Oneway ANOVA was adopted to test the statistically significant 
Table 2 Quantitative analysis in MNV subtypes (VT cutoff 8.40).

\begin{tabular}{|c|c|c|c|c|c|c|}
\hline \multirow[t]{2}{*}{ Parameter } & \multicolumn{2}{|l|}{ AMD } & \multicolumn{2}{|l|}{$\mathrm{CSC}$} & \multicolumn{2}{|l|}{ BVMD } \\
\hline & Group 1 & Group 2 & Group 1 & Group 2 & Group 1 & Group 2 \\
\hline Number of patients & 44 & 22 & 11 & 7 & 10 & 4 \\
\hline Age & $\begin{array}{l}78 \pm 8 \\
p>0.05\end{array}$ & $78 \pm 8$ & $\begin{array}{l}60 \pm 11 \\
p>0.05\end{array}$ & $63 \pm 11$ & $\begin{array}{l}26 \pm 10 \\
p>0.05\end{array}$ & $26 \pm 15$ \\
\hline $\begin{array}{l}\text { Speckled fluorescence } \\
\text { (quadrants) }\end{array}$ & $\begin{array}{l}1.05 \pm 1.03 \\
p=0.01^{*}\end{array}$ & $1.68 \pm 0.95$ & $\begin{array}{l}1.64 \pm 0.92 \\
p>0.05\end{array}$ & $2.14 \pm 0.69$ & $\begin{array}{l}0.0 \pm 0.0 \\
p>0.05\end{array}$ & $0.0 \pm 0.0$ \\
\hline Leakage area & $\begin{array}{l}3.69 \pm 3.28 \\
p>0.05\end{array}$ & $5.12 \pm 5.55$ & $\begin{array}{l}3.37 \pm 1.80 \\
p>0.05\end{array}$ & $4.34 \pm 2.12$ & $\begin{array}{l}2.80 \pm 1.48 \\
p>0.05\end{array}$ & $2.14 \pm 0.64$ \\
\hline MNV area & $\begin{array}{l}2.41 \pm 2.86 \\
p>0.05\end{array}$ & $3.79 \pm 4.24$ & $\begin{array}{l}1.39 \pm 1.50 \\
p>0.05\end{array}$ & $1.74 \pm 1.31$ & $\begin{array}{l}0.89 \pm 0.52 \\
p>0.05\end{array}$ & $1.08 \pm 0.35$ \\
\hline MNV/leakage ratio & $\begin{array}{l}0.59 \pm 0.31 \\
p<0.001 *\end{array}$ & $0.81 \pm 0.24$ & $\begin{array}{l}0.36 \pm 0.21 \\
p>0.05\end{array}$ & $0.40 \pm 0.15$ & $\begin{array}{l}0.32 \pm 0.10 \\
p=0.01 *\end{array}$ & $0.55 \pm 0.24$ \\
\hline LogMAR BCVA baseline & $\begin{array}{l}0.61 \pm 0.70 \\
p=0.03^{*}\end{array}$ & $0.28 \pm 0.25$ & $\begin{array}{l}0.29 \pm 0.20 \\
p>0.05\end{array}$ & $0.39 \pm 0.17$ & $\begin{array}{l}0.18 \pm 0.17 \\
p=0.02 *\end{array}$ & $0.40 \pm 0.08$ \\
\hline LogMAR BCVA 1-year & $\begin{array}{l}0.24 \pm 0.23 \\
p<0.001 *\end{array}$ & $0.45 \pm 0.33$ & $\begin{array}{l}0.18 \pm 0.16 \\
p=0.03^{*}\end{array}$ & $0.36 \pm 0.18$ & $\begin{array}{l}0.18 \pm 0.17 \\
p=0.003 *\end{array}$ & $0.60 \pm 0.27$ \\
\hline CMT baseline & $\begin{array}{l}423 \pm 88 \\
p>0.05\end{array}$ & $378 \pm 130$ & $\begin{array}{l}411 \pm 110 \\
p=0.02 *\end{array}$ & $297 \pm 51$ & $\begin{array}{l}419 \pm 85 \\
p>0.05\end{array}$ & $377 \pm 99$ \\
\hline CMT 1-year & $\begin{array}{l}360 \pm 76 \\
p>0.05\end{array}$ & $325 \pm 74$ & $\begin{array}{l}242 \pm 39 \\
p>0.05\end{array}$ & $208 \pm 59$ & $\begin{array}{l}319 \pm 66 \\
p>0.05\end{array}$ & $289 \pm 32$ \\
\hline CT Baseline & $\begin{array}{l}272 \pm 40 \\
p=0.03 *\end{array}$ & $206 \pm 69$ & $\begin{array}{l}368 \pm 22 \\
p=0.02 *\end{array}$ & $270 \pm 43$ & $\begin{array}{l}336 \pm 43 \\
p>0.05\end{array}$ & $288 \pm 56$ \\
\hline CT 1-year & $\begin{array}{l}263 \pm 34 \\
p=0.03 *\end{array}$ & $181 \pm 70$ & $\begin{array}{l}334 \pm 41 \\
p=0.01 *\end{array}$ & $244 \pm 44$ & $\begin{array}{l}323 \pm 56 \\
p>0.05\end{array}$ & $277 \pm 64$ \\
\hline HLT baseline & $\begin{array}{l}216 \pm 45 \\
p>0.05\end{array}$ & $174 \pm 68$ & $\begin{array}{l}289 \pm 23 \\
p>0.05\end{array}$ & $235 \pm 39$ & $\begin{array}{l}268 \pm 37 \\
p>0.05\end{array}$ & $257 \pm 69$ \\
\hline HLT 1-year & $\begin{array}{l}209 \pm 35 \\
p=0.04 *\end{array}$ & $162 \pm 52$ & $\begin{array}{l}259 \pm 54 \\
p>0.05\end{array}$ & $220 \pm 38$ & $\begin{array}{l}248 \pm 54 \\
p>0.05\end{array}$ & $244 \pm 60$ \\
\hline SLT baseline & $\begin{array}{l}55 \pm 7 \\
p<0.001 *\end{array}$ & $32 \pm 11$ & $\begin{array}{l}79 \pm 16 \\
p<0.001 *\end{array}$ & $35 \pm 5$ & $\begin{array}{l}68 \pm 13 \\
p<0.001 *\end{array}$ & $31 \pm 10$ \\
\hline SLT 1-year & $\begin{array}{l}54 \pm 5 \\
p<0.001 *\end{array}$ & $19 \pm 8$ & $\begin{array}{l}75 \pm 25 \\
p<0.001 *\end{array}$ & $24 \pm 9$ & $\begin{array}{l}75 \pm 13 \\
p<0.001 *\end{array}$ & $33 \pm 8$ \\
\hline N. intravitreal injections & $\begin{array}{l}7.2 \pm 1.5 \\
p<0.001 *\end{array}$ & $5.1 \pm 1.9$ & $\begin{array}{l}5.91 \pm 2.74 \\
p>0.05\end{array}$ & $6.14 \pm 3.08$ & $\begin{array}{l}0.30 \pm 0.95 \\
p<0.001^{*}\end{array}$ & $3.25 \pm 1.26$ \\
\hline VT MNV baseline & $\begin{array}{l}7.78 \pm 0.45 \\
p<0.001 *\end{array}$ & $9.75 \pm 1.39$ & $\begin{array}{l}7.26 \pm 0.77 \\
p>0.001 *\end{array}$ & $9.21 \pm 0.63$ & $\begin{array}{l}6.91 \pm 0.53 \\
p<0.001 *\end{array}$ & $9.18 \pm 0.64$ \\
\hline Vdisp MNV baseline & $\begin{array}{l}30.54 \pm \\
13.90 \\
p>0.05\end{array}$ & $\begin{array}{l}28.48 \pm \\
12.18\end{array}$ & $\begin{array}{l}19.80 \pm 4.81 \\
p>0.05\end{array}$ & $20.99 \pm 1.76$ & $\begin{array}{l}23.96 \pm 4.37 \\
p<0.001^{*}\end{array}$ & $51.09 \pm 8.87$ \\
\hline VD SCP baseline & $\begin{array}{l}0.37 \pm 0.02 \\
p>0.05\end{array}$ & $0.37 \pm 0.03$ & $\begin{array}{l}0.38 \pm 0.03 \\
p>0.05\end{array}$ & $0.38 \pm 0.03$ & $\begin{array}{l}0.39 \pm 0.03 \\
p>0.05\end{array}$ & $0.36 \pm 0.03$ \\
\hline VD DCP baseline & $\begin{array}{l}0.37 \pm 0.03 \\
p=0.03^{*}\end{array}$ & $0.36 \pm 0.02$ & $\begin{array}{l}0.36 \pm 0.02 \\
p>0.05\end{array}$ & $0.35 \pm 0.03$ & $\begin{array}{l}0.38 \pm 0.02 \\
p>0.05\end{array}$ & $0.37 \pm 0.01$ \\
\hline VD CC baseline & $\begin{array}{l}0.46 \pm 0.03 \\
p>0.05\end{array}$ & $0.45 \pm 0.03$ & $\begin{array}{l}0.46 \pm 0.04 \\
p>0.05\end{array}$ & $0.46 \pm 0.03$ & $\begin{array}{l}0.47 \pm 0.02 \\
p>0.05\end{array}$ & $0.47 \pm 0.04$ \\
\hline
\end{tabular}

differences between the parameters assessed in the three groups. Post-hoc analyses were performed to evaluate the differences between the VT-related subgroups, and Bonferroni correction was applied to assess for multiple comparisons. Tau-Kendall correlation analysis was used to investigate the relationship among the variables considered. The statistical significance was set at $p \leq 0.01$. The main outcome measurements were: BCVA, CMT, exudation thickness, VD of capillary plexa, VT and Vdisp of MNV, SF quadrants, leakage area, MNV area, and MNV/leakage ratio.

\section{Results}

Overall, 98 patients were included in the study, which included a 1-year follow-up. More specifically, 66 patients 

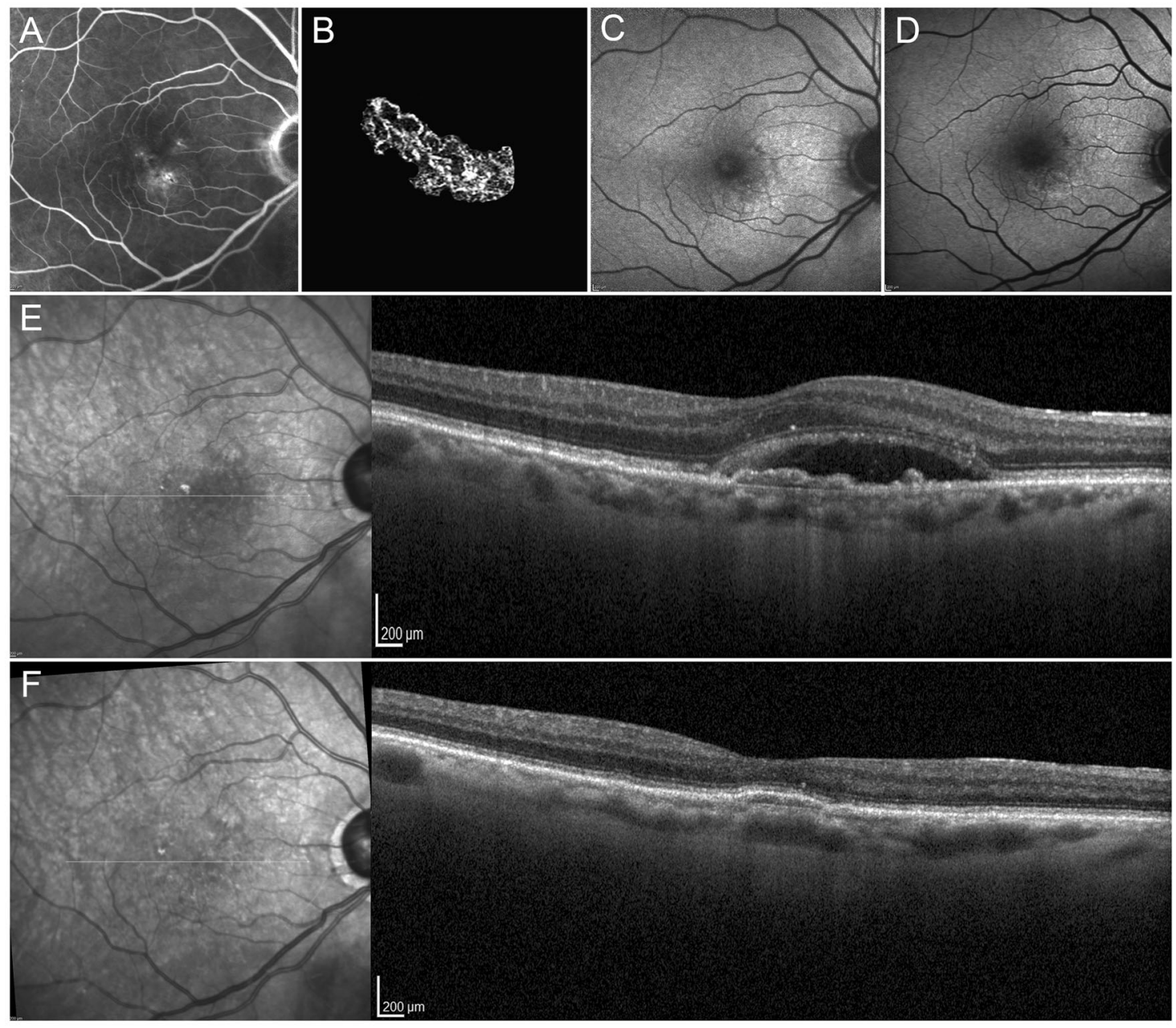

Fig. 1 Group 1 AMD-related MNV (MNV VT < 8.40). FA showed a marked leakage surrounding the MNV lesion in the late phase (A). OCTA reconstruction of the MNV is shown in $(\mathbf{B})$. Baseline FAF (C) revealed minor macular changes, almost unvaried after 1 year (D).

(66 eyes) were affected by AMD-related MNV (34 males; mean age $77 \pm 7$ years), 18 patients (18 eyes) suffered from CSC-related MNV (12 males; mean age $61 \pm 11$ years), and 14 patients (14 eyes) had BVMD-related MNV (12 males; mean age $21 \pm 12$ years), (10 eyes in Stage 2; 4 eyes in Stage 3). Clinical and imaging data are reported in Table 1. The ICC was 0.95 overall, while globally we registered 0.93 reproducibility and 0.89 repeatability of the measurements performed.

BCVA was alike in the three groups, both at baseline and after 1 year $(p>0.05)$. CMT was similarly comparable at baseline $(p>0.05)$; however, it proved to be greater in AMD patients than in CSC and BVMD patients after 1 year $(p<0.01)$. More speckled fluorescence quadrants were involved in CSC than in AMD $(p<0.01)$, whereas BVMD showed no speckled fluorescence. AMD patients revealed significantly greater leakage and a larger MNV area, as well as a higher MNV/leakage ratio, compared with CSC and
Baseline structural OCT (E) clearly detected a marked subretinal exudation; after 1 year $(\mathbf{F})$, the exudation was found to have been almost completely reabsorbed, without the onset of retinal atrophy.

BVMD patients $(p<0.01)$. MNV VT values were significantly higher in AMD than in CSC and BVMD $(p<$ 0.01 ), while CSC eyes displayed the lowest MNV Vdisp values $(p<0.01)$. VD in SCP and CC did not differ among the three groups $(p>0.05)$, whereas BVMD eyes had higher DCP VD values than AMD and CSC eyes $(p<0.01)$. The number of intravitreal injections differed among the three groups, being comparable in AMD and CSC $(p>0.05)$ but significantly lower in BVMD $(p<0.01)$.

We stratified all MNV patients according to an MNV VT cutoff of 8.40 in Group 1 (VT < 8.40) and Group 2 (VT > 8.40). AMD patients showed more speckled fluorescence quadrants in Group 2 than in Group $1(p<0.01)$, whereas no significant difference was found in CSC and BVMD. The leakage area and MNV area did not differ in Groups 1 and 2 in all three diseases $(p>0.05)$, whereas the MNV/ leakage ratio proved to be significantly higher in Group 2 of both AMD and BVMD eyes $(p<0.01)$. 

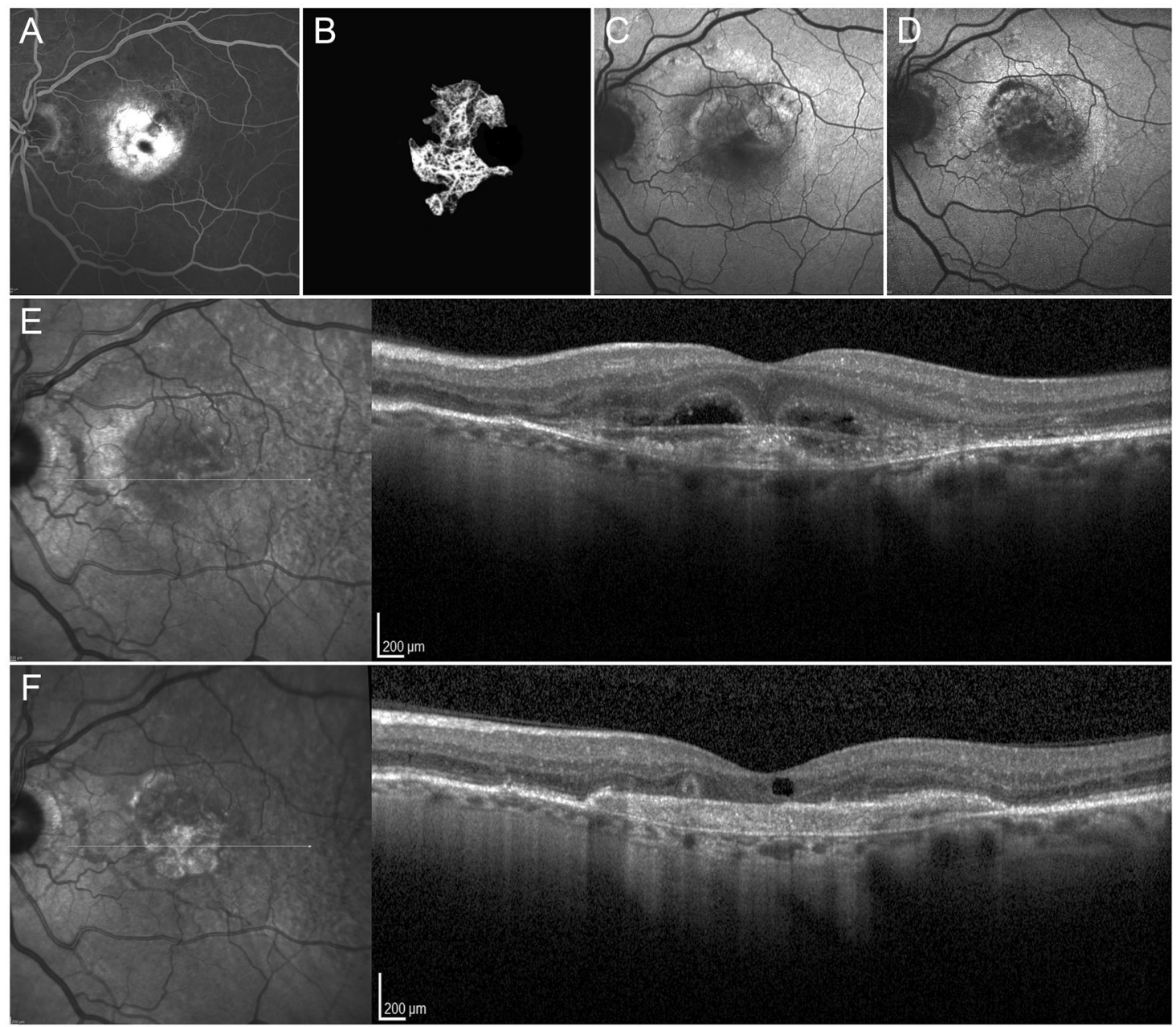

Fig. 2 Group 2 AMD-related MNV (MNV VT > 8.40). FA showed poor leakage in the late phase, with respect to the hyperfluorescent neovascular lesion (A). OCTA reconstructed MNV is shown in (B). Baseline FAF $(\mathbf{C})$ revealed diffuse and sparse autofluorescent changes over the entire posterior pole, with remarkable worsening after 1 year

Baseline BCVA was found to be significantly worse in Group 1 AMD eyes than in similar eyes in Group $2(p<$ 0.01 ), whereas with regard to BVMD eyes, BCVA was better in Group 1 than in Group $2(p<0.01)$. At the end of the follow-up, BCVA proved to be significantly better in Group 1 than in Group 2 in all three diseases $(p<0.01)$. CMT displayed unremarkable changes in Group 1 and Group 2 in the three diseases, both at baseline and at the end of the follow-up.

The choroid was significantly thinner in Group 2 than in Group 1. Moreover, our analysis revealed that SLT was the main contributor to choroidal thinning.

Significantly more intravitreal injections were performed in Group 1 than in Group 2 AMD eyes $(p<0.01)$. CSC eyes received a similar number of treatments. It is worth noting that in BVMD all Group 2 patients' eyes required intravitreal injections, whereas just one Group 1 eye, with the
(D). Baseline structural OCT (E) detected a well-defined subretinal hyporeflective lesion, with poor exudation; after 1 year $(\mathbf{F})$, the exudation was found to be absent, but structural OCT revealed the presence of retinal atrophy.

highest MNV VT value (7.80) in the group, required three anti-VEGF injections.

Only in BVMD eyes did we register significantly higher Vdisp values in Group 2 than in Group 1.

VD values in all three diseases were similar in Groups 1 and 2, with the sole exception of DCP VD in AMD eyes, where the worst values were found in Group $2(p=0.03)$. All the values are extensively reported in Table 2. Even though both CC VD and CT were similar in all groups, SLT was significantly thinner in Group 2 compared with Group $1(p<0.01)$.

The proportion of outer retinal atrophy detected after 1 year was as follows: $0 \%$ in Group 1 and $23 \%$ in Group 2 for AMD eyes $(p<0.01) ; 25 \%$ in Group 1 and $30 \%$ in Group 2 for CSC ( $p>0.05)$; $0 \%$ in Group 1 and $100 \%$ in Group 2 for BVMD $(p<0.01)$. Specimen cases of Group 1 and Group 2 AMD, CSC and BVMD eyes are shown in Figs. 1-6. 

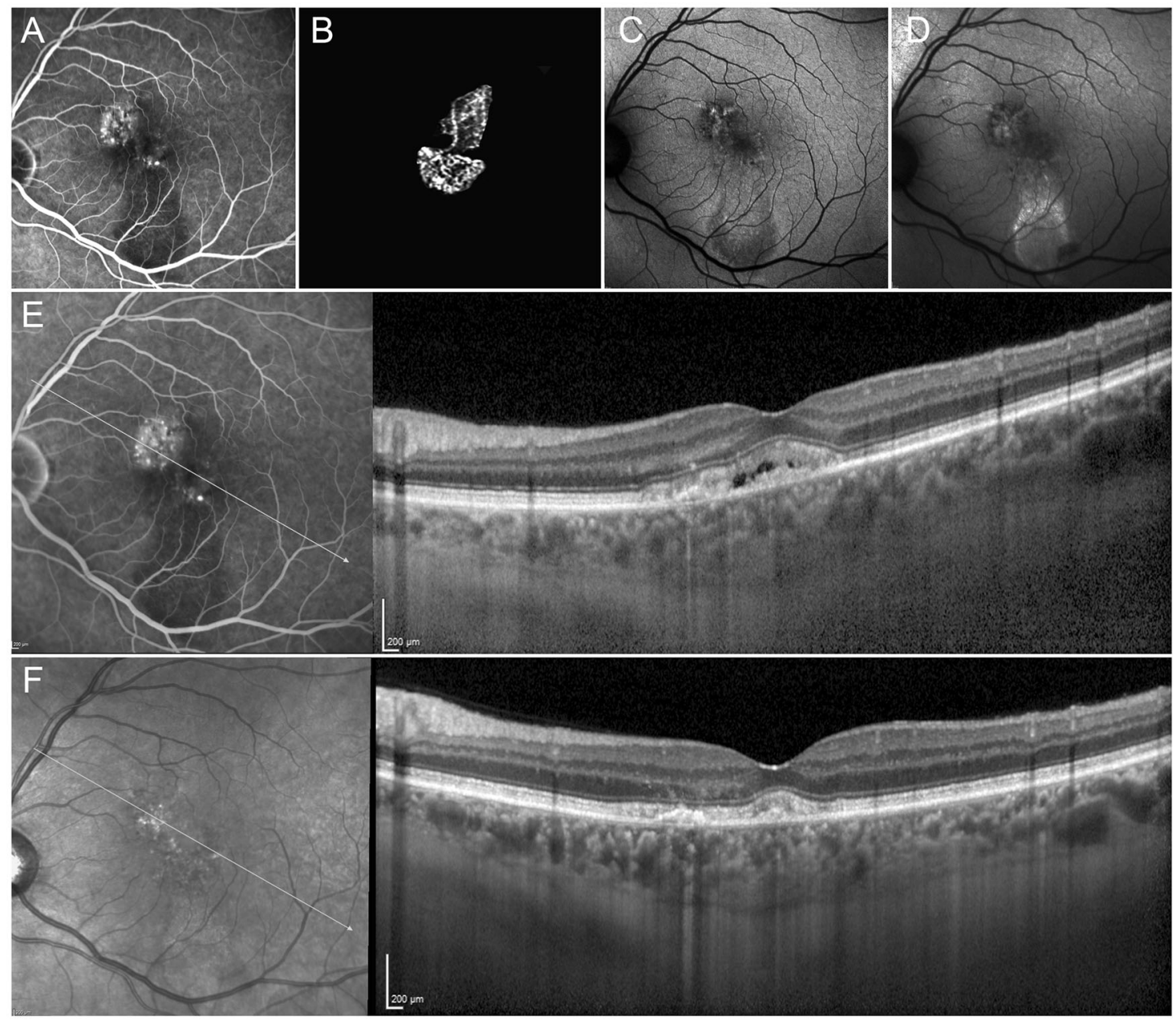

Fig. 3 Group 1 CSC-related MNV (MNV VT $<8.40$ ). FA showed a hyperfluorescent lesion surrounded by leakage in the late phase, in the context of CSC-related epithelial defects (A). Baseline FAF (B) revealed sparse macular changes, perfectly matching the 1-year

The correlation analysis revealed that higher MNV VT values are associated with less exudative phenomena and thinner SLT, but also with worse visual outcome in all three diseases (Supplementary Table 1).

\section{Discussion}

MNV complicates AMD, CSC, and BVMD in approximately $15 \%, 10 \%$, and $6 \%$ of cases, respectively [7-9]. The common pathogenetic factor is the abnormal upregulation and release of VEGF, leading to the development of a neovascular network and exudation. Regardless of the disease with which it is associated, MNV responds well to anti-VEGF injections; [9-11] moreover, MNV secondary to CSC may benefit from PDT, even in combined therapy [12, 13]. Despite MNV sharing a pathogenetic origin with other diseases, it is not clear to what extent follow-up $(\mathbf{C})$. Baseline structural OCT $(\mathbf{D})$ showed subretinal exudation, which was completely reabsorbed after 1 year $(\mathbf{E})$, without the onset of retinal atrophy.

it might have different morphological and functional features.

In the present study, we adopted a quantitative OCTAbased approach to identify different MNV subtypes in three macular diseases characterized by different pathogenic mechanisms. The first finding of interest was that an OCTA quantitative cutoff based on MNV VT was indeed effective in categorizing MNV eyes in clinically different subgroups.

With regard to MNV secondary to AMD, we found that lesions displaying higher MNV VT were characterized by less exudative manifestations (reduced CMT and reduced leakage) and thinner choroid, and more frequently evolved towards outer retinal atrophy. The overall findings suggest that there may be two main MNV subforms. The variant with lower MNV VT is distinguished by a higher VEGF expression and release, as supported by greater exudation indices (CMT and MNV/leakage ratio), and responds well to anti-VEGF treatment administered PRN on the basis of 

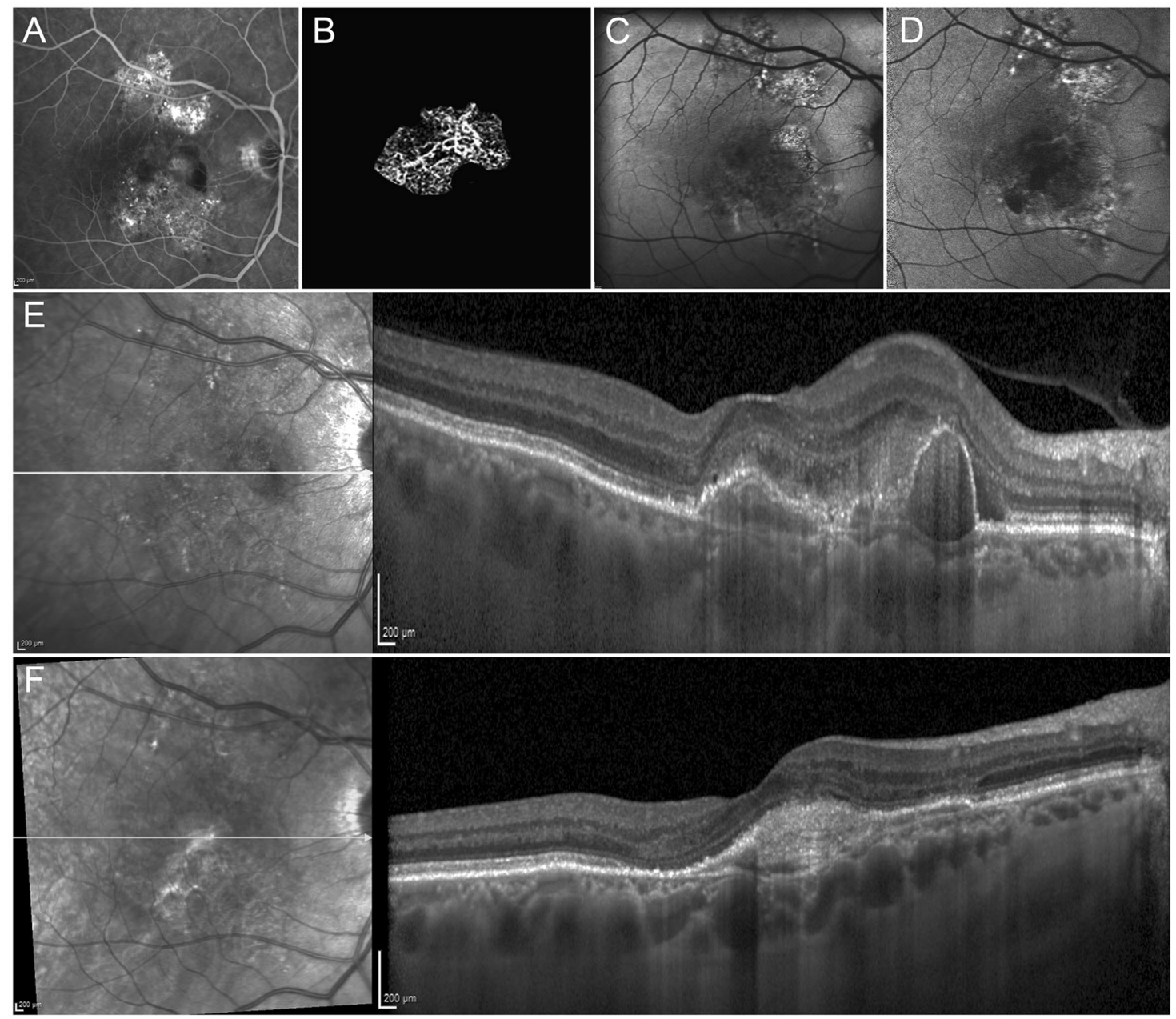

Fig. 4 Group 2 CSC-related MNV (MNV VT > 8.40). FA detected the MNV in the context of diffuse alterations localized over the entire posterior pole (A). OCTA shows up the neovascular lesion well (B). These changes, well highlighted by the baseline FAF image (C), proved to have worsened after 1 year, when a marked reduction in autofluorescence was detected (D) baseline structural OCT (E) detected a big subretinal hyporeflective lesion, with almost no exudative phenomena; after 1 year $(\mathbf{F})$, structural OCT recorded a notable shrinking of the hyporeflective lesion, without exudation but with evident atrophic changes. the fluid detected, giving rise to fewer atrophic changes, while the form featuring higher MNV VT and reduced exudative manifestations may be less dependent on VEGF release and more frequently evolves to atrophy. This latter subform required fewer anti-VEGF injections following a PRN regimen based on the detection of fluid/leakage, possibly owing to a more complex pathogenesis that also involves other cytokines and growth factors, ultimately causing a wider RPE and choroidal impairment. MNV in Group 2 notably displayed a diminished SLT compared with MNV in Group 1.

MNV VT can differentiate two subforms of MNV secondary to CSC, leading to different final visual outcomes. Interestingly, there was a negative correlation between $\mathrm{MNV}$ VT and CMT: the higher the MNV VT, the lower the CMT, in accordance with the pattern described in AMD-related MNV. Atrophic changes developed in about $1 / 3$ of cases in both CSC subforms, possibly due to the administration of PDT. Higher MNV VT was inversely associated with leakage in BVMDrelated MNV, suggesting a more stable subtype of MNV.

It is worth noting that low level of damage was displayed by Group $1 \mathrm{MNV}$ in BVMD eyes.

Remarkably, the present findings regarding the applicability of an MNV VT cutoff of 8.40 to categorize two clinically relevant subgroups of MNV lesions matched the conclusions of two recently published papers applying the same cutoff in AMD-related MNV [5, 6]. In these two previous studies, this VT cutoff value enabled MNV to be categorized according to the amount of perfusion determining the MNV VT value, not distinguishing between the different types of MNV lesions (type 1, type 2, and mixed MNV).

The present study extended the results of these two previous investigations by testing this MNV VT cutoff value in MNV secondary to different retinal diseases. Our findings 

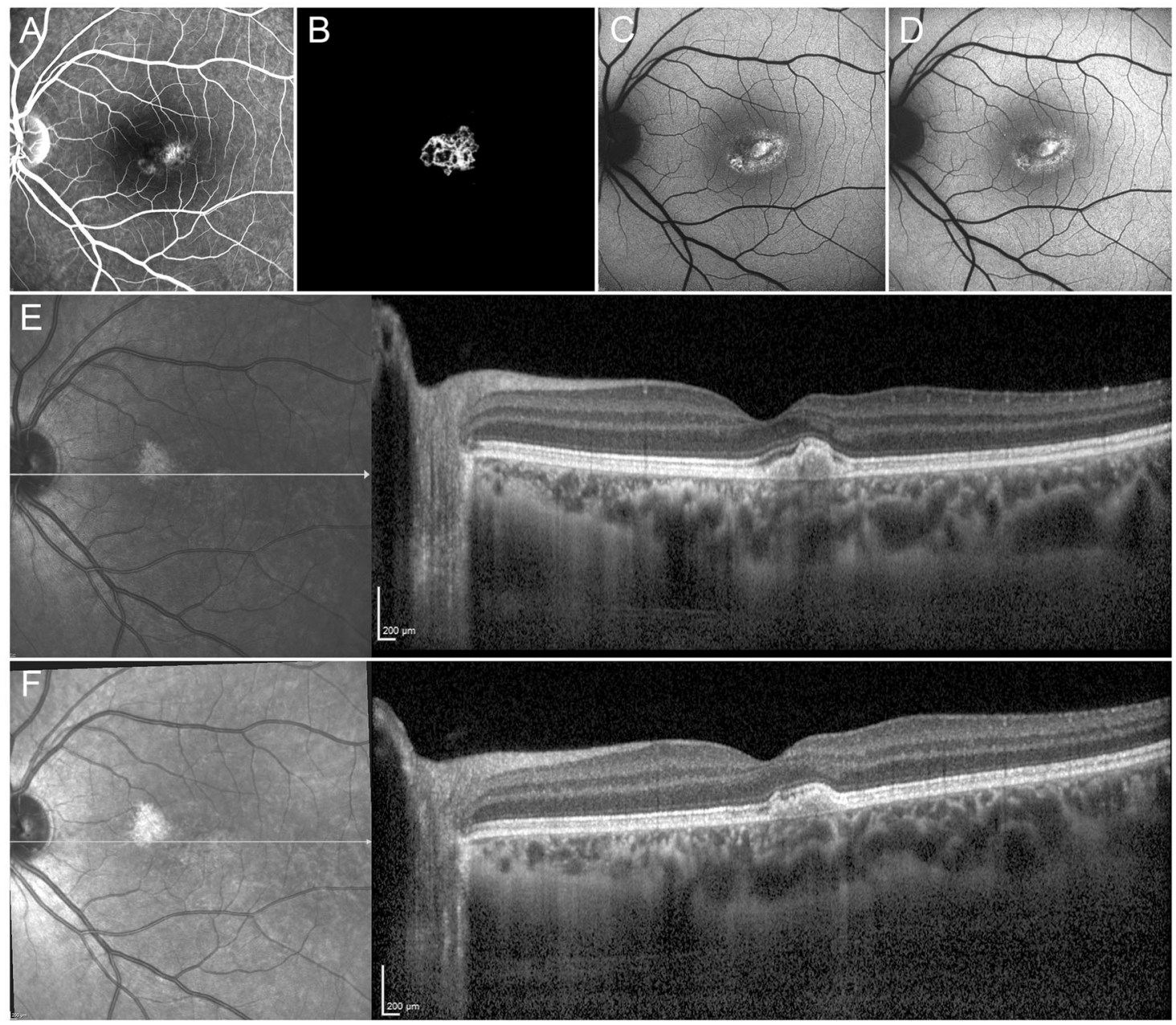

Fig. 5 Group 1 BVMD-related MNV (MNV VT < 8.40). FA showed a clear hyperfluorescent lesion surrounded by leakage (A). OCTA clearly reveals a neovascular network (B). Baseline FAF (C) revealed hyper- and hypo-fluorescent macular changes, almost unchanged after

suggested that MNV VT is a parameter mainly related to the amount of blood flow perfusion, i.e., the MNV activity, and is less affected by the type of lesion and the specific retinal disease leading to the onset of this complication. Like previous investigations [5, 6], this MNV VT cutoff value made it possible to gather patients into two distinct clusters that did not display features associated with a continuum of numbers. From this point of view, this proposed MNV VT cutoff, if confirmed by more extensive investigations, might achieve sufficient relevance to be applied in standard clinical practice and thus provide a reliable basis for scheduling personalized treatment strategies.

In all three diseases, we registered a significantly thinner choroid in Group 2 than in Group 1. This finding agreed with previous studies showing that a thinner choroid is associated with the development of retinal atrophy [14]. Remarkably, we found SLT to be most altered in Group 2, suggesting that the thinning of the Sattler layer might be
1 year (D). Baseline structural OCT $(\mathbf{E})$ detected a hyperreflective subretinal lesion, without exudation; after 1 year $(\mathbf{F})$, the lesion was found to have become smaller, without exudation and without the need for anti-VEGF treatment over the entire follow-up.

responsible for the reduced blood supply to the outer retina - a phenomenon most evident in Group 2 MNVs thus leading to more atrophic changes.

We are aware that our study labors under several limitations, mainly related to the relatively small number of patients and the short follow-up. Moreover, all multimodal imaging techniques may have been affected by artifacts. Our findings and hypotheses certainly need to be backed up by larger prospective evaluations and histological confirmation. Lastly, we chose AMD, CSC, and BVMD as diseases representing three different pathological conditions, mainly related to the aging process, primary functional alterations in middle-aged patients, and genetically determined dysfunctions, respectively. We excluded other maculopathies showing MNV complications, above all pathologic myopia, owing to their higher level of multimodal imaging artifacts or insufficient prevalence. Future studies should include all retinal diseases complicated by MNV. 

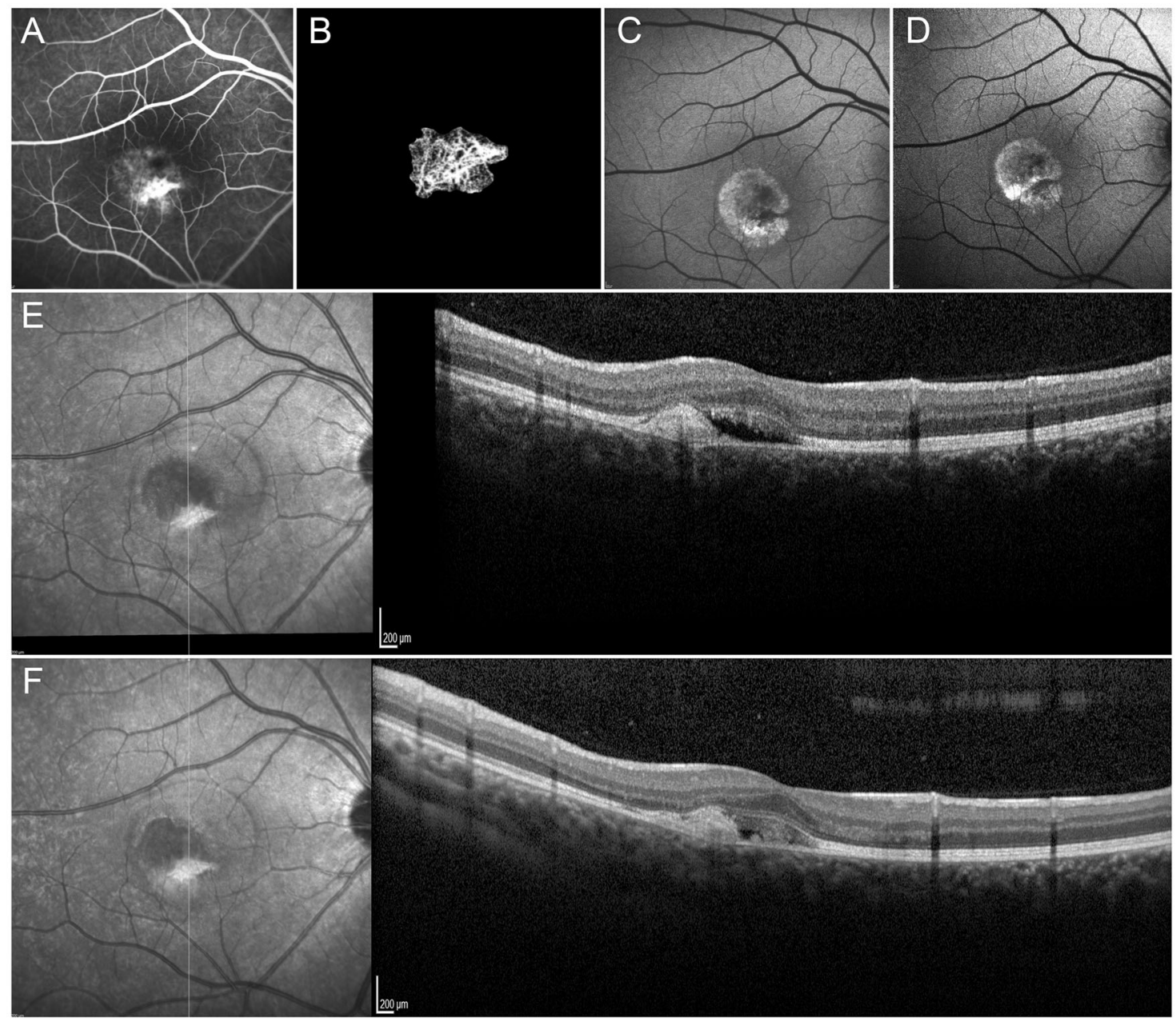

Fig. 6 Group 2 BVMD-related MNV (MNV VT > 8.40). FA showed a macular MNV with leakage and epithelial defects (A). The MNV was well-reconstructed by OCTA (B). Baseline FAF (C) revealed diffuse changes in autofluorescence, with considerable worsening after

In conclusion, our study highlighted features common to $\mathrm{MNV}$ lesions developed in AMD, CSC, and BVMD, as well as differences between them. Significantly, a quantitative OCTA approach, rather than qualitative evaluations, proved to offer a step forward in the stratification of MNV in clinically relevant subgroups in all the diseases considered. We suggest our findings offer a solid foundation for future clinical trials and the differences detected might be helpful in reaching an optimal, personalized treatment.

\section{Summary}

\section{What was known before}

- OCTA is a feasible technique to study macular neovascularization. The adoption of an even more advanced
1 year (D). Baseline structural OCT $(\mathbf{E})$ detected a hyperreflective lesion in the context of subretinal exudation; after 1 year $(\mathbf{F})$, the exudation had diminished, but structural OCT found ellipsoid zone disruption in the region of the lesion.

quantitative approach may provide steps forward to reach new clinically relevant information.

\section{What this study adds}

- MNV can be categorized into two clinically relevant subgroups, differing in the final outcome. The adoption of the same quantitative OCTA cutoff resulted useful in MNV secondary to macular diseases characterized by completely different pathogenesis, namely AMD, CSC, and best vitelliform macular dystrophy.

\section{Compliance with ethical standards}

Conflict of interest FB is a consultant for Alcon (Fort Worth, Texas, USA), Alimera Sciences (Alpharetta, Georgia, USA), Allergan Inc. 
(Irvine, California, USA), Farmila-Thea (Clermont-Ferrand, France), Bayer Shering-Pharma (Berlin, Germany), Bausch And Lomb (Rochester, New York, USA), Genentech (San Francisco, California, USA), Hoffmann-La-Roche (Basel, Switzerland), Novagali Pharma (Évry, France), Novartis (Basel, Switzerland), Sanofi-Aventis (Paris, France), Thrombogenics (Heverlee, Belgium), Zeiss (Dublin, USA). All other authors declare that they have no conflict of interest.

Publisher's note Springer Nature remains neutral with regard to jurisdictional claims in published maps and institutional affiliations.

\section{References}

1. Green WR, Wilson DJ. Choroidal neovascularization. Ophthalmology. 1986;93:1169-76.

2. Spaide RF, Jaffe GJ, Sarraf D, Freund KB, Sadda SR, Staurenghi $\mathrm{G}$, et al. Consensus nomenclature for reporting neovascular agerelated macular degeneration data: consensus on neovascular agerelated macular degeneration nomenclature study group. Ophthalmology. 2020;127:616-36.

3. Patel R, Wang J, Campbell JP, Kiang L, Lauer A, Flaxel C, et al. Classification of choroidal neovascularization using projectionresolved optical coherence tomographic angiography. Invest Ophthalmol Vis Sci. 2018;59:4285-91.

4. Arrigo A, Aragona E, Capone L, Pierro L, Romano F, Bandello F, et al. Advanced optical coherence tomography angiography analysis of age-related macular degeneration complicated by onset of unilateral choroidal neovascularization. Am J Ophthalmol. 2018;195:233-42.

5. Arrigo A, Romano F, Aragona E, Di Nunzio C, Battista M, Bandello F, et al. Optical coherence tomography angiography can categorize different subgroups of choroidal neovascularization secondary to age-related macular degeneration. Retina. 2020; 40:2263-69.
6. Arrigo A, Aragona E, Di Nunzio C, Bandello F, Parodi MB. Quantitative optical coherence tomography angiography parameters in type 1 macular neovascularization secondary to age-related macular degeneration. Transl Vis Sci Technol. 2020;9:48.

7. Jager RD, Mieler WF, Miller JW. Age-related macular degeneration. N Engl J Med. 2008;358:2606-17.

8. Lee GI, Kim AY, Kang SW, Cho SC, Park KH, Kim SJ, et al. Risk factors and outcomes of choroidal neovascularization secondary to central serous chorioretinopathy. Sci Rep. 2019;9:3927.

9. Khan KN, Mahroo OA, Islam F, Webster AR, Moore AT, Michaelides M. Functional and anatomical outcomes of choroidal neovascularization complicating BEST1-related retinopathy. Retina. 2017;37:1360-70.

10. Solomon SD, Lindsley K, Vedula SS, Krzystolik MG, Hawkins BS. Anti-vascular endothelial growth factor for neovascular agerelated macular degeneration. Cochrane Database Syst Rev. 2019; 3:CD005139.

11. Chhablani J, Pichi F, Silva R, Casella AM, Murthy H, Banker A, et al. Antiangiogenics in choroidal neovascularization associated with laser in central serous chorioretinopathy. Retina. 2016;36:901-8.

12. Lu HQ, Wang EQ, Zhang T, Chen YX. Photodynamic therapy and anti-vascular endothelial growth factor for acute central serous chorioretinopathy: a systematic review and meta-analysis. Eye (Lond). 2016;30:15-22.

13. Daruich A, Matet A, Dirani A, Bousquet E, Zhao M, Farman N, et al. Central serous chorioretinopathy: recent findings and new physiopathology hypothesis. Prog Retin Eye Res. 2015;48:82-118.

14. Fan W, Abdelfattah NS, Uji A, Lei J, Ip M, Sadda SR, et al. Subfoveal choroidal thickness predicts macular atrophy in agerelated macular degeneration: results from the TREX-AMD trial. Graefes Arch Clin Exp Ophthalmol. 2018;256:511-8. 